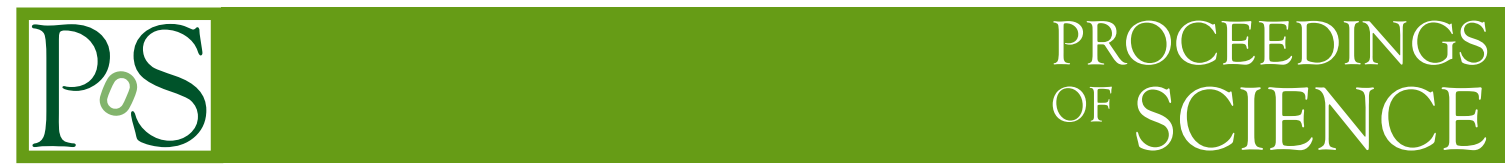

\title{
High and very high energy gamma-ray emission from binaries
}

\section{Guillaume Dubus*}

Laboratoire d'Astrophysique de Grenoble, UMR 5571 CNRS, Université Joseph Fourier, BP 53, 38041 Grenoble, France

Emission from binaries above several $\mathrm{MeV}$ has long being terra incognita. Gamma-ray lore speaks of near-encounters with stars behaving mysteriously in faraway energy bands. Very high energy emission from several binaries is now been well established and as surprising as those early tales. I will review the current observational status, focusing on the very high energies. I will then discuss how such emission might arise in these systems, the associated difficulties, and what can be learned from such observations.

VII Microquasar Workshop: Microquasars and Beyond September 1-5 2008

Foca, Izmir, Turkey

\footnotetext{
* Speaker.
} 


\section{Introduction: terra incognita}

The first attemps to detect very high energy light from astrophysical sources via atmospheric Cherenkov radiation date back at least 50 years (Weekes, 2007). Yet, the road has been long, fraught with uncertainties, false alerts and dead ends as the techniques required for these difficult observations matured. But surprising discoveries laid in wait, ready for the picking. Observations by the HESS, MAGIC and VERITAS collaborations have now established close to a hundred sources of very high energy gamma-ray radiation, light with energies $\geq 10^{11} \mathrm{eV}$ (Horns, 2008). Of these, four (actually $3+0.5+0.5$, see $\S 2$ ) have been identified with binaries composed of a massive star and a compact object, neutron star or black hole.

The frequency space above $1 \mathrm{MeV}$ is no longer terra incognita. There is as much frequency space between a $1 \mathrm{GHz}$ radio photon and $1 \mathrm{keV} \mathrm{X-ray} \mathrm{photon} \mathrm{than} \mathrm{there} \mathrm{is} \mathrm{between} \mathrm{a} 10 \mathrm{keV}$ photon and a $1 \mathrm{TeV}\left(10^{12} \mathrm{eV}\right)$ very high energy gamma-ray photon. Non-thermal processes necessarily dominate at such high energies. Exploring this new frontier can therefore bring new insights into phenomena that we suspect or know to be associated with non-thermal processes such as relativistic ejections from compact objects. The vastness of this frequency space has the same allure than the last terra incognita: the Antarctic. White, barren and empty at first sight compared to more familiar surroundings, closer examination reveals a rich ecology, subtle physical processes and unscathed traces of past history that are essential (if not crucial) to understand the more 'temperate' regions.

The next section reports on the recent landings on this terra australis of the electromagnetic spectrum in the context of compact binaries $(\$ 2)$. The information collected is then grouped to propose a geography of these systems $(\S 3)$. Simple recipes to navigate these faraway lands are given in $\$ 4$ before concluding.

\section{Land ahead!}

The present age of exploration could not happen without the newest generation of sturdy, reliable instruments and analysis techniques in the VHE $\left(>10^{11} \mathrm{eV}\right)$ domain. HESS, MAGIC and VERITAS allow localization of point sources to sub-arcminute precision and investigations of variability on one-day timescales for $0.1 \mathrm{Crab}$ sources. These have proved to be the breakthrough performances to firmly associate gamma-ray emission with binaries.

\subsection{Gamma-ray binaries}

- PSR B1259-63. The first binary to have been detected at VHE energies is PSR B1259-63. A 48-ms radio pulsar discovered in a radio survey, it is in a 3.5 year orbit around a Be star. The radio pulse is eclipsed around periastron, which is also associated with radio flaring and a high X-ray flux. VHE emission had been expected to occur near periastron passage (Kirk et al., 1999) and was duly found by the HESS collaboration (Beilicke et al. 2004; Aharonian et al. 2005b; this was also the first time two VHE sources were seen in the same field-of-view - a few degrees - the other source, HESS J1303-631, is still unidentified). VHE emission has also been detected at the last periastron passage in late 2007. 
- LS 5039. HESS discovered this source in its survey of the inner Galactic Plane (Aharonian et al., 2005a). LS 5039 first appears in a cross-correlation of ROSAT sources with O/B stars (Motch et al., 1997). It was then singled-out from high-mass X-ray binaries due to its radio emission $(<10 \%$ of catalogued $\mathrm{HMXBs}$ have this property), this being resolved on milliarcsecond scales, making the source a microquasar candidate (Paredes et al., 2000). EGRET source 3EG1824-1514 is coincident with LS 5039 but, with an error box of $\sim 0.5$ and no detected variability, the association remained tentative. The HESS position excludes the other potential counterparts to the 3EG source. In addition, the VHE flux is strongly modulated on the 3.9 day orbital period: indeed, a blind search of the VHE observations recovers the orbital frequency with an accuracy comparable to that of the radial velocity optical studies (Aharonian et al., 2006).

- LS I +61303. This Be + compact object binary has long history of associations with gammaray sources. Almost 30 years ago, Cos B observations detected a source whose error box contained this high mass X-ray binary with intriguing periodic radio flares (Hermsen et al., 1977; Gregory et al., 1979). However, the positional accuracy and lightcurve from the gamma-ray source were unable to provide a firm association — even with EGRET and even though this is one of the brightest sources in the $>100 \mathrm{MeV}$ sky. MAGIC was able to detect the source, confirmed by VERITAS, and prove that its VHE flux is modulated on the 26 day orbital period (Albert et al., 2006; Acciari et al., 2008; Albert et al., 2008b).

- [HESS J0632+057]. This source was detected serendipitously by HESS in the Galactic Plane. It is the only point-like unidentified HESS source. It is coincident with a Be star (no information on a possible binary nature). Recent observations report a variable X-ray counterpart consistent with the Be star location (Hinton et al., 2008). The overall spectral energy distribution (SED) is reminiscent of the other binaries described above, making this likely to be the first binary identified through its VHE gamma-ray emission. This is to be confirmed, hence this counts only as a half-detection.

The latter two highlight a key aspect of these binaries: bar their gamma-ray emission, these are quite inconspicuous objects at other wavelengths and that probably explains why it took some time to establish their outstanding character. In fact, plotting their spectral energy distributions shows these binaries emit most of their non-stellar output beyond $1 \mathrm{MeV}$ - hence the name gamma-ray binaries (Dubus, 2006a). Their discovery is, at least to this author, quite a surprise. This terra australis is far from empty and, indeed, carries its own landmarks not easily seen from afar. The recent launch of the Fermi Gamma-ray Space Telescope, surveying the sky above $>100 \mathrm{MeV}$ with a factor 30 improvement in sensitivity over EGRET, carries high hopes for the detection of further gamma-ray binaries (Dubois, 2008).

\subsection{Cyg X-1}

Cyg X-1 deserves special mention. Unlike all the above, Cyg X-1 is a well-established black hole candidate (actually the first one) and microquasar, with all the usual phenomenology associated with accreting sources. Like the above, it has a massive star counterpart (O type) and is one of the HMXBs with radio emission. The MAGIC collaboration reported a detection of VHE emission 
from this source during one night, part of a month long monitoring campaign. When taking into trial factors, the detection significance is $4.1 \sigma$ (Albert et al., 2007). The ratio $L_{\mathrm{vhe}} / L_{\mathrm{X}}$ is $\sim 10^{-4}$ when it is $\sim 1$ in the gamma-ray binaries above.

It is fair to say that this detection is not considered secure by the VHE community. Single, low significance detections during flares have achieved notoriety due to the past history of the field (Weekes, 1992). The VHE detection gains credence from the fact that this occurred at the time of a 3-day long X-ray high flux state, unprecedented at least in the INTEGRAL timeline. However, this has to be mitigated since, looking at the INTEGRAL lightcurve in Malzac et al. (2008), MAGIC sees no VHE emission at the time of highest X-ray flux. Naturally, expecting a one-to-one correspondence between both bands could be simplistic - but then the coincidence of the 3 day X-ray flare with the MAGIC detection cannot be used as supporting evidence.

Added complications come when taking into (naive?) expectations. One would be that $\gamma$-ray emission is associated with X-ray spectral state changes from hard to soft, when it is conjectured (on the basis of observations) that the binary emits discrete relativistic ejecta. Cyg X-1 stayed in the low/hard X-ray state all throughout its high flux episode. There is no easy association to make. Furthermore, the VHE detection occurred at a time when the black hole was behind its O star companion as seen by the observer. VHE gamma-rays, if emitted close to the black hole, would have had to go through the whole radiation field of the companion. The VHE photon and stellar photons have sufficient energies to produce pairs, thereby completely absorbing the VHE emission. Avoiding this absorption requires the VHE source to be $\geq 0.7$ a.u. from the black hole, above the orbital plane (Bosch-Ramon, 2008a). This is interestingly close to the size of the compact jet resolved in radio VLBI observation — but there is as yet no model to account for such emission.

At this stage the conclusion must be that this source should be confirmed (counting as half a detection in §1). The best hope is Fermi which has the sensitivity and all-sky monitoring abilities to detect a one-day flare from this object (Dubus, 2007).

\section{Where do we stand?}

All of the binaries detected in (very) high energy gamma-rays harbour massive stars and display radio emission, resolved on milliarsecond scales in the case of LS I +61 303 , LS 5039 and Cyg X-1. The massive star must bear some responsibility in the VHE emission: either by providing a large amount of material via its wind (exerting a pressure on any outflow from the compact object and target protons for hadronic high energy interactions); and/or because it is a generous source of seed photons for inverse Compton scattering.

\subsection{Asia!}

Christopher Columbus on his initial voyage assumed he had landed somewhere in West India based on his knowledge of faraway lands (India, China). Working the analogy, the resolved radio maps of LS 5039 and LS I +61³03 suggest these sources power relativistic jets like the established 
microquasars. This is rather natural: it is fact that relativistic jets contain highly energetic particles. Those are detected via their emission, extending from radio all the way to $\mathrm{X}$-rays in a few cases (XTE J1550-564, H1743-322). This implies electrons with TeV energies, enough to produce VHE gamma-ray emission at some level (likely very low in the previous two cases, see Dubus 2007). Models of how VHE emission could arise in such jets have been duly proposed by several groups (Atoyan \& Aharonian, 1999; Georganopoulos et al., 2002; Romero et al., 2003; Bosch-Ramon et al., 2006; Bednarek, 2006). The models typically inject power-laws of particles in a structured jet and compute the expected emission from synchrotron, inverse Compton (external on the star, or internal on synchrotron) and decays from hadronic interactions (Romero, 2008).

\subsection{America!}

There are several issues with this interpretation, and one wonders if we have not stumbled upon a new land (albeit, like America, already visited by a few intrepid early explorers !). In a microquasar, one would expect signatures of accretion processes (outbursts, rapid variability, $\mathrm{Fe}$ lines), radio and X-ray spectral changes, relativistic motion. LS I +61 303 and LS 5039 show mild variability compared to accreting sources, optically thin radio spectra, hard X-ray spectra with no apparent cutoff before $100 \mathrm{keV}$. Their overall behaviour and spectra are much more reminiscent of PSR B1259-63 than of microquasars. Indeed, these are gamma-ray binaries with high $L_{\mathrm{vhe}} / L_{\mathrm{x}}$. Taking the cues from PSR B1259-63, an appealing conjecture is that all gamma-ray binaries are young pulsars with massive companions (Dubus, 2006a), an interpretation that had originally been suggested for LS I+619303 in a prescient paper by Maraschi \& Treves (1981).

High $B$ field, fast rotating young neutron stars generate relativistic winds with sufficient pressure to inhibit accretion. These are stopped in their expansion by pressure from the strong stellar wind and confined to a comet-like shape if the ratio of pulsar wind to stellar wind momentum flux is low. Particle acceleration at the shock generate the high-energy emission(Maraschi \& Treves, 1981; Tavani \& Arons, 1997; Kirk et al., 1999; Sierpowska \& Bednarek, 2005; Dubus, 2006a; Khangulyan et al., 2007; Sierpowska-Bartosik \& Torres, 2007), progressively cooling down as they are carried away in the tail, emitting radio.

Gamma-ray binaries are a new class of young binaries, progenitors of high-mass X-ray binaries. As the pulsar slows down and its rotation energy is dissipated (with about $1 \%$ ending up as VHE emission, as seems to be the case in pulsar wind nebulae powered by isolated young pulsars), pressure from the relativistic wind will become insufficient to stop accretion and an accreting X-ray pulsar will be turn on. The lifetime of gamma-ray binaries is short compared to HMXBs but population synthesis calculations find there should be of order 100 in our Galaxy at present (Portegies Zwart \& Verbunt, 1996).

\subsection{Binary pulsar wind nebulae}

This interpretation is supported by radio VLBI observations. In the last Microquasar Workshop, Dhawan et al. (2006) reported on a month long campaign covering a full orbit of LS I+61 303 . The position angle of the source is shown to rotate with orbital phase in a similar fashion to what 
would be expected from a cometary tail. There is no detected relativistic motion and lower frequency emission (cooler particles) occurs further down the tail. The radio morphology at a given orbital phase appears reproducible (Albert et al., 2008a). VLBA observations reported by Moldón (2008) suggest similar behaviour in LS 5039.

Such observations may be used to better understand the interaction geometry. Figure 1 shows the observations of LS I $+61^{\circ} 303$ (Dhawan et al., 2006). The expected shock location between the pulsar and stellar wind, and the initial direction of the comet tail are superposed. The latter is obtained by adding the stellar wind and pulsar orbital vector motions and projecting the resulting vector on the sky with an inclination of $60^{\circ}$. The pulsar wind is assumed to have a power of $10^{36}$ $\mathrm{erg} \mathrm{s}^{-1}$ (as PSR B1259-63). The result if the pulsar interacts in the plane of the slow, dense equatorial wind of the Be star is shown on the left. Right panel shows interaction with the fast, tenuous polar wind. In the latter case, the shock is far from the pulsar (and unlikely to be collimated, see Romero et al. 2003) and the vectors do not fit. On the other hand, the agreement is reasonable for an interaction with the equatorial wind.

In addition to constraining the interaction between the winds, high resolution radio observations could be used to constrain the magnetic field and cooling in the radio tail and the magnetisation of the pulsar wind $\left(\sigma=B^{2} /\left(4 \pi \Gamma n_{e} m_{e} c^{2}\right)\right)$. Close to the pulsar the dynamics are set by the magnetic field and $\sigma$ is high. Close to the shock, observations of pulsar wind nebulae like the Crab suggest $\sigma \ll 1$ to explain the non-thermal emission. How one goes from a high to a low $\sigma$ is not understood. In gamma-ray binaries the shock location is typically at 0.1 a.u. $=10^{4} R_{L}$ (where $R_{L}$ is the light cylinder radius) compared to $10^{9} R_{L}(=0.1 \mathrm{pc})$ in a PWN like the Crab, much closer to the pulsar. Hence, gamma-ray binaries offer interesting prospects to address the $\sigma$-problem. A simple 1D model of the comet tail shows that the expected step of the spiral created as the pulsar tail wraps up around the companion is $\sigma c P_{\text {orb }}$ : a measure of this step would constrain $\sigma$ (for details and caveats see Dubus 2006a). Comparison with numerical simulations of the interaction should bear fruit in the coming years (Bogovalov et al., 2008).

\subsection{Settling (the matter) ?}

Unfortunately, detecting a pulse will be difficult for lack of photons and a precise ephemeris in gamma-rays and because of free-free absorption due to the stellar wind in radio (as seen in PSR B1259-63). Hence, bar the exceptional case of PSR B1259-63 with its long orbit, proving that these gamma-ray binaries are powered by a pulsar is likely to be indirect.

An interesting piece of evidence is the recent detection of a magnetar-like burst by the Swift BAT from within 88" of LS I+61³03 (Barthelmy et al., 2008; Dubus \& Giebels, 2008). The burst lasted $230 \mathrm{~ms}$, released $10^{37} \mathrm{ergs}$ and is best fitted by a blackbody of $7.5 \mathrm{keV}$ and radius $\approx 100 \mathrm{~m}$ (at $2.3 \mathrm{kpc}$ ). Plugging in those numbers implies $B \geq 10^{15} \mathrm{G}$ (see $\$ 2.2$ in Thompson \& Duncan 1995). Quenching accretion (assumed Eddington) with such a magnetic field implies a pulsar power $L_{p} \geq 510^{36} B_{15}^{-2 / 7} \dot{M}_{18}^{8 / 7} \mathrm{erg} \mathrm{s}^{-1}$ or, equivalently, a pulse period $P<1.5 B_{15}^{4 / 7} \dot{M}_{18}^{-2 / 7}$ seconds: a young magnetar. This raises several puzzles. What about previous burst activity? Where is the magnetar quiescent emission? Where are the X-ray pulsations? How did a magnetar form in a 


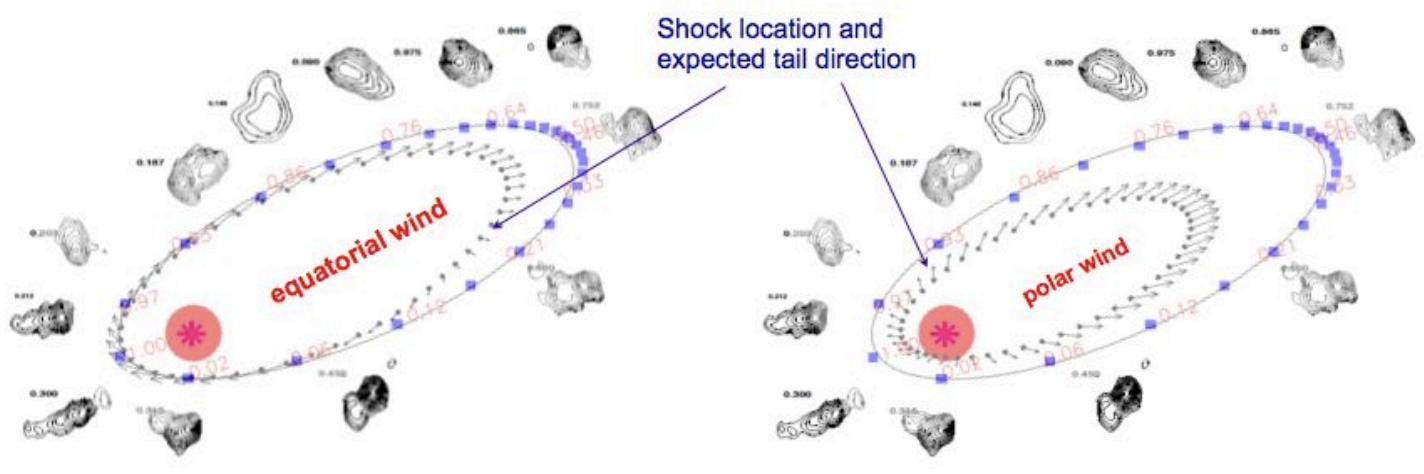

Figure 1: Montage of radio VLBI observations of LS I +61\%303 (Dhawan et al., 2006). Superposed is the expected shock location between the pulsar and stellar wind, and the initial direction of the comet tail.

binary? Magnetars are very young stars: where is the supernova remnant ? Marti et al. (1998) find extended radio emission around LS I+61 303 and conclude a SNR origin is unlikely as this would make the remnant anomalously underluminous.

Observations reported even more recently show LS I+61 303 has short flares in X-rays with an implied size $\leq 610^{10} \mathrm{~cm}$ (Smith et al., 2008). This is the size of the shocked region in the case of interaction with an equatorial wind at periastron (Dubus, 2006a): wind clumps may cause fluctuations in the emission and structure of the shocked region. More puzzling is the telegram of Ray \& Hartman (2008) reporting Z source-like fast variability in a single RXTE observation of the LSI field a few weeks before the burst. Several sources in the same field-of-view may be toying with us (a LMXB Z-type neutron star, a magnetar and LS I+61 303 ?!) but if all are related to LS I then this would imply that we have an object that sometimes accrete (not unlikely if the spin period has decreased to a few seconds, the threshold to stop accretion given above).

\section{Navigating the faraway frequency lands}

All models, regardless of the particular interpretation, use the same basic ingredients to explain the emission, purely leptonic models having the public's favour because of their simplicity (hadronic interactions are casually set aside here, and the interested reader is referred to Romero 2008). Here are the basic processes required to navigate the treacherous straits when mapping the coast of these faraway frequency lands.

\subsection{Particle acceleration}

Obviously a spout of particle acceleration is required for the electrons to reach the $\mathrm{TeV}$ energies required. One goal of such observations is indeed to investigate under what conditions this occurs and the resulting distribution. Tactfully passing over the details of the acceleration, all models assume some simple injection (power-law) of electrons up to some maximal energy consistent 
with radiative losses. Obtaining this may require a dash of nuclei. Acceleration must be extremely efficient in these systems for the electrons to reach the required energies (Khangulyan et al., 2008). The emitting distribution can be located close to the compact object (shocked or unshocked pulsar wind, jet) or further away (Bosch-Ramon, 2008a).

\subsection{Geometrical constrains}

Observations of binaries allow measurements of stellar masses and radii. A binary brings strict geometrical and dynamical constraints to bear on models of the physical processes at work. In a binary, the pulsar wind interacts with the stellar wind of its companion in a periodic fashion. The binary nature constraints the matter and radiation densities sampled by the pulsar during its orbital motion.

As an illustration, looking at the steady-state distribution obtained by injecting power-law distribution of electrons in the radiation field of the companion star and in a uniform magnetic field, it can be shown that the electron spectrum in gamma-ray binaries develops a characteristic break at the energy where synchrotron losses dominate inverse Compton losses (Dubus, 2006a). Inverse Compton losses are inefficient at high energies because of the reduced cross-section in the KleinNishina regime. This gives two break frequencies: one in X-rays (synchrotron radiation) and one in the VHE (inverse Compton) domain. In this simple approach, the first depends only on the binary parameters whereas the second depends also on the magnetic field $\varepsilon_{\mathrm{IC}}=4\left(R_{10} T_{4} / d_{0.1}\right) B_{1}^{-1} \mathrm{TeV}$ where $R$ is the stellar radius in units of $10 R_{\odot}, \mathrm{T}$ is the star temperature in units of $40000 \mathrm{~K}$, and the orbital separation $d$ is in units of 0.1 a.u. Provided the simple assumptions are met, measuring the VHE cutoff gives the magnetic field.

\subsection{Inverse Compton on an anisotropic seed photon field}

The inverse Compton cross-section has a dependence on the geometry: the interaction depends on the angle between the incoming photon and electron direction (Dubus et al., 2008; Khangulyan et al., 2008). For instance, the limit between the Thomson and Klein-Nishina regime is given by $\varepsilon \gamma(1-\beta \cos \psi)=m_{e} c^{2}$ where $\varepsilon$ is the energy of the photon, $\gamma$ is the Lorentz factor of the electron and $\psi$ is the angle between the two incoming directions. When both travel in the same direction $(\psi \approx 0)$ the interaction occurs in the Thomson regime. However, the number of scatterings is low. On the other hand, when the two travel in opposite directions $(\psi \approx \pi)$ the interaction occurs deep in the Klein-Nishina regime and there is a lot of scatterings (e.g. driving on the wrong side of the motorway).

Even if the distribution of electron speeds is isotropic, because of the strong relativistic beaming of the emission from a very energetic electron, an observer sees only those few electrons whose vector happens to be aligned to the line-of-sight (Fig. 2). At superior conjunction this electron is moving towards the star, hence produces a strong (scatterings) soft (Klein Nishina) spectrum. At inferior conjunction this electron is moving away from the star, producing a hard (Thomson) but low (few scatterings) spectrum. Hence, everything else being equal, a modulation of the high 


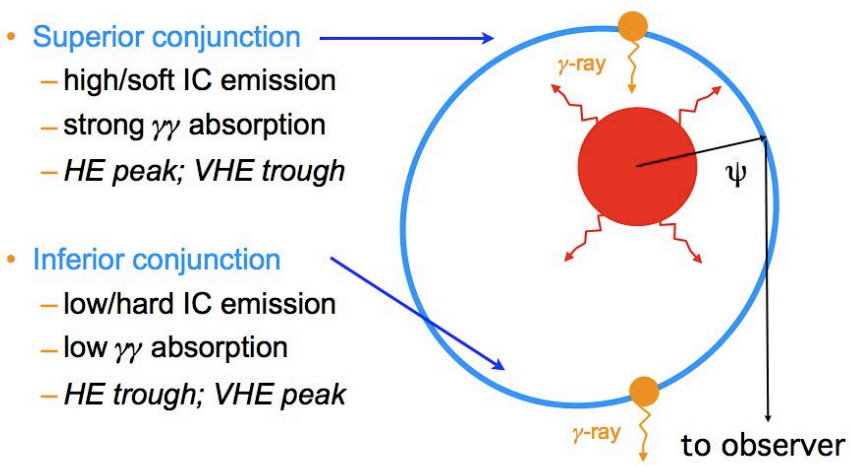

Figure 2: Summary of the impact of the radiative processes along the orbit in a gamma-ray binary.

energy gamma-ray emission is still expected because of the anisotropic nature of inverse Compton radiation if the dominant source of seed photons is the massive star (as very likely to be the case considering its huge radiative output).

\subsection{VHE gamma-ray absorption and pair production}

VHE photons with energies above the threshold $\varepsilon_{\min }=60(1-\cos \psi)^{-1} T_{4}^{-1} \mathrm{GeV}$ can pair create on stellar photons (Moskalenko, 1995; Bednarek, 1997; Dubus, 2006b). The interaction produces a $e^{-} e^{+}$pair. If the interaction occurs far from the threshold one of the particles end up with most of the energy of the incoming VHE photon; at threshold the energy is split between the particles. This is also an angle dependent effect. If secondary emission from the particles can be neglected, then this is equivalent to absorption of the VHE flux. The effect is maximal at superior conjunction, when the gamma-ray has to go through the whole radiation field of the companion star. It is minimal at inferior conjunction. The impact on the spectral energy distribution is to create a trough above the threshold (the cross section drops away from the threshold).

In LS 5039 absorption should completely absorb the VHE flux at superior conjunction if it originated from the immediate vicinity of the compact object. However, HESS still detects some VHE photons. This implies that emission occurs further away (as discussed for Cyg X-1 in §2.2) or that secondary emission by the produced pairs acts to decrease the opacity. The pairs may spread around, fill up the binary system and produce a constant, low-level emission (Bosch-Ramon et al., 2008c). It is not clear yet whether this is feasible since it depends sensitively on the intensity of the magnetic field in the system - which acts to cool the particles via synchrotron emission before they can re-emit VHE photons (Bosch-Ramon et al., 2008b).

\subsection{Simple recipe for LS 5039}

A simple recipe to reproduce the LS 5039 gamma-ray emission above $100 \mathrm{MeV}$ is proposed in Dubus et al. (2008). This assumes steady injection of electrons with a power-law distribution in a region close to the compact object with a unifom magnetic field. The only modulation to expect is 
based only on the above geometrical considerations. HESS sees a high and a low VHE flux depending on orbital phase, with different spectra. Fitting the high state spectra imposes $B=0.8 \pm 0.2 \mathrm{G}$ (this sets the observed cutoff in the VHE spectrum), a power law index of $\alpha=-2 \pm 0.3$ (close to the canonical value) and a total energy in electrons of $310^{37}$ ergs (compatible with a pulsar wind interpretation).

Letting the system evolve along the orbit reproduces the HESS modulation and predicts a modulation in the Fermi range with a peak at superior conjunction. This is easily explained: Fermi observes roughly between $0.1-10 \mathrm{GeV}$, below the threshold for pair production. Hence, only the modulation due to anisotropic inverse Compton acts in this band. The peak IC emission occurs at superior conjunction, which happens to occur close to periastron in LS 5039. This is a robust prediction which is made by several models with different mixes of ingredients: the basic reason is the radiative processes which act in the same simple way in all of them (Bednarek, 2007; SierpowskaBartosik \& Torres, 2007; Dubus et al., 2008; Khangulyan et al., 2008; Torres, 2008). A default of this model is that it predicts no VHE flux at superior conjunction: as discussed in the previous section, this could be due to secondary pair emission.

\subsection{Simple recipe for $\mathrm{LS} \mathrm{I}+61^{\circ} 303$}

Reproducing the modulation at VHE energies in LS I+61³03 requires more complex modelling. The peak of the VHE emission occurs far from the companion star, close to apastron (at maximum distance). Absorption of VHE gamma-rays is not expected to play a major role in this system (Dubus, 2006b) and can certainly not explain why no VHE flux is seen at inferior conjunction where minimum absorption is expected and which also happens to be close to periastron: hence, a high VHE flux would be expected. Unlike LS 5039, the orbit of the compact object is more eccentric, causing large variations e.g. in the shock location between the pulsar and stellar winds.

As discussed in $\S 3.3$ (Fig 1), the pulsar is probably orbiting in the dense equatorial wind of its companion. This puts the shock location very close to the pulsar at periastron $\left(\approx 510^{10} \mathrm{~cm}\right.$, Dubus 2006a) and implies a high magnetic field, even more so if the neutron star is a magnetar. A field of $100 \mathrm{G}$ (plausible) is largely sufficient to forbid electrons from reaching the energies required for VHE emission. They will lose energy to synchrotron before they can. On the other hand, further away from the Be star, the pressure drops and the shock is further away from the pulsar with a lower magnetic field. There acceleration to TeV energies and VHE emission can occur (Dubus, 2006a). In any case, the same general considerations given above imply gamma-ray emission in the Fermi band should still peak close to superior conjunction (extra synchrotron emission will only add to that), i.e. close to periastron passage in LS I+61 303 . Here again an anti-correlation is expected.

\subsection{Emission from the free pulsar wind ?}

The pre-shock ('free') pulsar wind is usually described as a cold, mono-energetic and highly relativistic (bulk Lorentz factor $\Gamma \sim 10^{4}-10^{6}$ ) pair plasma. The magnetic field is frozen in the plasma and no synchrotron emission is expected. In PWN the pre-shock zone is dark. However, in 
binary PWN the free pulsar wind can inverse Compton scatter radiation from the massive star. Calculations show that with standard values of $\dot{E}$ and $\Gamma$ this results in a very intense inverse Compton line that is excluded by observations (Khangulyan et al., 2007; Cerutti et al., 2008). Actually, it may turn out that the free pulsar wind actually dominates the gamma-ray emission and not the shocked wind, as proposed by Sierpowska-Bartosik \& Torres (2007) (see Torres 2008 in these proceedings). In this case, more complex particle distributions are required than simple mono-energetic distributions.

A seductive alternative is that the pulsar wind remains highly magnetized up to the shock. In that case, there would be no kinetic energy for the particles to radiate. There is some support for this idea. In the striped pulsar wind model, magnetic fields of opposite polarities are separated by a wavelike current sheet (Kirk et al., 2007). The strong magnetic field can be dissipated in this sheet and give kinetic energy to the particles. However, it needs time to do this. The wavelength of the sheet is equal to the light cylinder radius and, since dissipation cannot occur faster than the speed of light, the timescale on which this occurs is at least $\Gamma R_{L} / c$ in the proper frame. However, if the shock location is too close then there is not enough time for dissipation. This sets a maximum bulk Lorentz factor above which no dissipation occurs $\Gamma<\left(R_{S} / R_{L}\right)^{1 / 2}$ (Arons, 2008) which is $\approx 100$ for gamma-ray binaries. This is very stringent and models usually suggest the initial $\Gamma$ is greater than a 100. Hence, there could be no dissipation of the striped pulsar wind before the shock in gamma-ray binaries. Particle acceleration may nevertheless still occur as the $B$ field dissipates behind the shock (Pétri \& Lyubarsky, 2007).

\section{Conclusion}

- With HESS, MAGIC, VERITAS, the recent launch of Fermi and future projects like AGIS and CTA, high-energy and very high energy gamma-ray astronomy has entered a golden age. Surprises await.

- HE and VHE emission from binaries is now established beyond all doubt.

- This emission in fact dominates the non-stellar output in gamma-ray binaries. These are likely to be powered by rotational spindown of a young pulsar (magnetar?). These are binary pulsar wind nebulae.

- Interaction with the massive star generates all sorts of interesting constrained stuff, notably (1) a radio comet tail, (2) the creation of matter from light, (3) a modulation of the gamma-ray emission.

- It is possible to infer physical properties about the pulsar wind and provide a fresh look on a 30 year old problem: the formation of relativistic winds from rotating, highly magnetized pulsars.

- In setting sail for the terra australis of the electromagnetic spectrum we have landed on the gamma-ray binary archipelago. The microquasar promised land may be within sight. 


\section{Acknowledgments}

It is a pleasure to thank the organisers of this conference for their invitation and their support. I acknowledge support from the European Community via contract ERC-StG-200911

\section{References}

[1] Acciari, V. A., et al. (VERITAS collaboration) 2008, ApJ, 679, 1427

[2] Albert, J., et al. (MAGIC collaboration) 2008, ApJ, 684, 1351

[3] Albert, J., et al. (MAGIC collaboration) 2008, ArXiv e-print 0806.1865

[4] Albert, J., et al. (MAGIC collaboration) 2007, ApJ, 665, L51

[5] Albert, J., et al. (MAGIC collaboration) 2006, Science, 312, 1771

[6] Aharonian, F., et al. (HESS collaboration) 2006, A\&A, 460, 743

[7] Aharonian, F., et al. (HESS collaboration) 2005, Science, 309, 746

[8] Aharonian, F., et al. (HESS collaboration) 2005, A\&A, 442, 1

[9] Arons, J. 2008, in 40 Years of Pulsars: Millisecond Pulsars, Magnetars and More, AIP Conf. Proc. 983, 222

[10] Atoyan, A. M., \& Aharonian, F. A. 1999, MNRAS, 302, 253

[11] Barthelmy, S. D., et al. 2008, GCN Circular 8215

[12] Bednarek, W. 2007, A\&A, 464, 259

[13] Bednarek, W. 2006, MNRAS, 368, 579

[14] Bednarek, W. 1997, A\&A, 322, 523

[15] Beilicke, M., Ouchrif, M., Rowell, G., \& Schlenker, S. 2004, The Astronomer's Telegram, 249,1

[16] Bogovalov, S. V., et al. 2008, MNRAS, 387, 63

[17] Bosch-Ramon, V., VII Microquasar Workshop, PoS (MQW7) 070

[18] Bosch-Ramon, V., Khangulyan, D., \& Aharonian, F. A. 2008, arXiv:0808.1540

[19] Bosch-Ramon, V., Khangulyan, D., \& Aharonian, F. A. 2008, A\&A, 482, 397

[20] Bosch-Ramon, V., Romero, G. E., \& Paredes, J. M. 2006, A\&A, 447, 263

[21] Cerutti, B., Dubus, G., \& Henri, G. 2008, A\&A, 488, 37 
[22] Dhawan, V., Mioduszewski, A., \& Rupen, M. 2006, VI Microquasar Workshop, POS (MQW6) 052

[23] Dubois, R. 2008, VII Microquasar Workshop, PoS (MQW7) 019

[24] Dubus, G., \& Giebels, B. 2008, The Astronomer's Telegram, 1715, 1

[25] Dubus, G., Cerutti, B., \& Henri, G. 2008, A\&A, 477, 691

[26] Dubus, G. 2007, The First GLAST Symposium, AIP Conf. Proc., 921, 65

[27] Dubus, G. 2006, A\&A, 456, 801

[28] Dubus, G. 2006, A\&A, 451, 9

[29] Georganopoulos, M., Aharonian, F. A., \& Kirk, J. G. 2002, A\&A, 388, L25

[30] Gregory, P. C., Taylor, A. R., Crampton, D. et al. 1979, AJ, 84, 1030

[31] Hermsen, W., Swanenburg, B. N., Bignami, G. F. et al 1977, Nature, 269, 494

[32] Hinton, J. A., et al. 2008, ApJ, accepted (arXiv:0809.0584)

[33] Horns, D. 2008, to appear in Reviews of Modern Astronomy (arXiv:0808.3744)

[34] Khangulyan, D., Aharonian, F., \& Bosch-Ramon, V. 2008, MNRAS, 383, 467

[35] Khangulyan, D., Hnatic, S., Aharonian, F., \& Bogovalov, S. 2007, MNRAS, 380, 320

[36] Kirk, J. G., Lyubarsky, Y., \& Petri, J. 2007, to appear in Springer Lecture Notes on "Neutron stars and pulsars, 40 years after the discovery", ed W.Becker, arXiv:astro-ph/0703116

[37] Kirk, J. G., Ball, L., \& Skjaeraasen, O. 1999, Astroparticle Physics, 10, 31

[38] Malzac, J., et al. 2008, A\&A, in press (arXiv:0805.4391)

[39] Maraschi, L., \& Treves, A. 1981, MNRAS, 194, 1P

[40] Marti, J., et al. 1998, A\&A, 329, 951

[41] Martocchia, A., Motch, C., \& Negueruela, I. 2005, A\&A, 430, 245

[42] Moldón, J. 2008, VII Microquasar Workshop, PoS (MQW7) 022

[43] Moskalenko, I. V. 1995, Space Science Reviews, 72, 593

[44] Motch, C., Haberl, F., Dennerl, K., Pakull, M., \& Janot-Pacheco, E. 1997, A\&A, 323, 853

[45] Paredes, J. M., Martí, J., Ribó, M., \& Massi, M. 2000, Science, 288, 2340

[46] Pétri, J., \& Lyubarsky, Y. 2007, A\&A, 473, 683

[47] Portegies Zwart, S. F., \& Verbunt, F. 1996, A\&A, 309, 179 
[48] Ray, P. S., \& Hartman, J. M. 2008, The Astronomer's Telegram, 1730, 1

[49] Romero, G. 2008, VII Microquasar Workshop, PoS (MQW7) 020

[50] Romero, G. E., Torres, D. F., Kaufman Bernadó, M. M., \& Mirabel, I. F. 2003, A\&A, 410, L1

[51] Sierpowska, A., \& Bednarek, W. 2005, MNRAS, 356, 711

[52] Sierpowska-Bartosik, A., \& Torres, D. F. 2007, ApJl, 671, L145

[53] Smith, A., et al. 2008, ApJ, submitted (arXiv:0809.4254)

[54] Tavani, M., \& Arons, J. 1997, ApJ, 477, 439

[55] Torres, D., VII Microquasar Workshop, PoS (MQW7) 021

[56] Thompson, C., \& Duncan, R. C. 1995, MNRAS, 275, 255

[57] Weekes, T. C. 2007, in Energy Budget in the High Energy Universe, Proceedings of the International Workshop held 22-24 February, 2006, in Kashiwa, Japan, World Scientific Publishing Co., p.282 (arXiv:astro-ph/0606130)

[58] Weekes T. 1992, Space Science Rev., 59, 315 